\title{
The Case for Wikis in Foreign Language Teaching
}

\author{
Xiaohui Sun, D.Ph. ${ }^{1}$, Branko Medic, D.Sc. ${ }^{2}$ \\ ${ }^{1}$ Weifang University \\ xiaohuisun@wfu.edu \\ ${ }^{2}$ Manchester University \\ medic.tesol@gmail.com
}

Article History: Received: 11 January 2021; Accepted: 27 February 2021; Published online: 5 April 2021

\section{Introduction}

Web 2.0 has immensely impacted language learning since the mid 2000s (Reinhardt, 2019). The term was coined by Tim O'Reilly in 2004 to suggest the evolution of the web to interactivity, collaboration and openness (Boulos and Wheelert, 2007). "Web 1.0 was commerce. Web 2.0 is people" (Singel, 2005), who are now active participants, instead of passive recipients of education.

Social media are the Web 2.0 applications that gained greatest popularity among foreign language students and professionals. Among them, wikis, blogs and social media are most influential for language teaching, and attracted considerable academic research (Reinhardt, 2019).

In this research paper, the focus is on wikis, as accessible and simplified means for collaboration (Reinhardt, 2019). For example, by using this collaborative technology, foreign language students are allowed to write a text together, define terms and concepts, brainstorm, revise the work of others, critically reflect, and many more (Kessler, 2009; Ducate, Anderson and Moreno, 2011). From learners' perspective, wikis are enjoyable, engaging, motivating and rewarding (Lund, 2008; Aydin and Yildiz, 2014; Wang, 2014). Yet, some scholars have raised issues about information accuracy (Ducate, Anderson and Moreno, 2001), participation resistance (Lund, 2008) and unequal contribution (Arnold, Ducate and Kost, 2009), which might make the use of wikis by educators problematic.

\subsection{Personal context}

While there is a debate on the wikis' effectiveness, the is one universally accepted idea: learners are the beneficiary of any teaching changes. Indeed, there is a call for a shift in education, since traditional lecturing is not effective. Research shows that students are mentally present $40 \%$ of the time during a teacher-centred class; and they are able to retain only $20 \%$ of the information by its end (McLeish, 1968). It is therefore puzzling why most teachers continue with traditional approaches. The personal motivation to study Web 2.0 technology is driven from the realworld need for improving knowledge processes. Personality traits such as conscientiousness, devotion and openness to experience motivate the formulation of research puzzles that bring critical reflection and effective change.

\subsection{Professional context}

Technological developments introduced new means for instructors to promote collaborative learning in foreign language courses (Aydin and Yildiz, 2014). Professionals are invited to utilise these new ways and make language material more relevant and engaging for students. Participatory Web 2.0 technologies like wikis seem to provide lucrative opportunities. However a wiki is only a social media platform, and merely using it in the vocational college does not automatically result in improved learning outcomes. Research shows that with no proper teacher intervention even the well-designed collaborative environments will likely fail (Alghasab, Hardman and Handley, 2019). It is not enough for a professional be aware on wikis and use them. For the needs of professional development, depth knowledge and critical thinking are required, in order to make the best out of wikis for the needs of foreign language learners.

\section{A review of available sources}

The term wiki comes from the phrase wiki-wiki, which in Hawaiian means quick (Reinhardt, 2019). Wikis first became popular among programmers as quick means for organising and sharing content in a collaborative manner (Reinhardt, 2019). Simply put, wikis are websites that make it possible for anyone to participate and edit (Ducate, 
Anderson and Moreno, 2001). Chase (2007) uses the beehive metaphor, as participants in wiki projects work together and divide labour to accomplish an objective and create something.

In the context of education, wikis are used by pre-defined groups as collaborative tools to edit each other's work (Alshumaimeri, 2011). Therefore, what distinguishes wikis is collaboration, with enhanced opportunities for interaction and autonomous language learning for improved course outcomes.

\subsection{The impact of wiki-supported on interaction}

The social cognitive perspective in teaching has recently attracted significant attention, since meaningful interactions are useful but also enjoyable for language learners (Aydin and Yildiz, 2014; Hudson, 2018). Still, the idea is not new. Sociocultural theories can be traced in Vygotsky's work (1978) and specifically the theory of zone of proximal development. Vygotsky (1978) states that interaction between pupils is essential for the successful completion of tasks, where more advanced students provide scaffolding to less knowledgeable peers.

For instance, Wang et al. (2013) highlight the benefits of increased bi-directional communication between native and non-native speakers. According to the scholars, the shared space of wikis facilitates authentic input, as well as meaning negotiations. The latter refers to solving communication problems, and not necessarily language errors. Further, negotiation is crucial for language teaching and learning since it recognises gaps in development. The platform itself amplifies such interactions, as wikis enliven and simplify dialogue and feedback provision (Huang and Huang, 2010).

To benefit from the interaction opportunities that wiki-supported pedagogy offers, tutors cannot only rely on providing the tool for co-authoring and discussion (Alghasab, Hardman and Handley, 2019). Some studies revealed students' resistance to interact and engage in wiki-enabled tasks, be it due to intergroup hierarchies, tensions between group and individual ownership, or just because of preferences for individual writing (Lund, 2008; $\mathrm{Li}$ and Zhu, 2013).

In this line of thought, Biasutti (2017) compares the use of forum and wikis in language learning. She rightly points out that wiki assignments are outcome-oriented, i.e. towards production and development. Meanwhile, interactions are not a particular strength of the tool. On the contrary, forums are more about communication. Given this, the structure, motivation and personal evaluation that the teacher provides is of utmost importance for the level of interaction in wiki groups ( $\mathrm{Li}$ and $\mathrm{Zhu}, 2013$ ).

\subsection{Impact on collaboration}

Moving on to collaboration, this suggests more refined and complex level of interaction (Bradley, Lindstrom and Rystedt, 2010). Collaboration is greatly emphasised in literature on wiki-enabled tutoring. Academics suggest that the process of knowledge construction in the context of foreign languages is based on co-construction with peers (Hauck and Youngs, 2008; Aydin and Yildiz, 2014). The greatest benefit of wikis, cited by Hudson's (2018) sample of foreign language learners, is namely collaboration.

When collaboration takes place, students are not only engaged in content product, but also in participating in the ideas of others, evaluating and reflecting on them (Bradley, Lindstrom and Rystedt, 2010). Such features allow wikis to drive more participants' involvement than other web-mediated environments (Wang, et al., 2014). For example, contrary to blogs, which put authorship in the forefront, wikis focus on collaborative content production, with socialisation and in discourse communities (Warschauer and Grimes, 2007).

Additionally, Pellet's results (2012) indicate a sense of community and engagement with study material, when collaboration takes place through a wiki. Similarly, Bradley, Lindstrom and Rystedt (2010) report that when collaboration was evident in their groups of software engineering students, learning English, they were more involved, making a higher number of edits and producing more text versions. Clearly, it appears that learners may also fail to collaborate, and "interaction does not categorically mean collaboration" (Donato, 2004, p. 285).

\subsection{Impact on autonomous learning}

When engaged in a collaborative wiki project, learners have a dual role: they are simultaneously authors and audience. They shift from passive recipients of foreign language material to knowledge producers and editors. Therefore, students are empowered with different roles, and the educational process is more democratic (Ducate, Anderson and Moreno, 2001).

In other words, wikis help educators facilitate an autonomous learning environment (Kessler, 2009) and thus, applying the principles of the learner-centred approach. Language learners are provided with means to work 
together without the leading presence of the instructor. Instead, they are encouraged to interact with each other, discuss ideas and edits, and overall, participate in collaborative learning (Cowan, et al.; Reo, 2006).

A particular strength of wikis is the possibility for teachers to offer a collaborative learning design, that is autonomous and can happen outside the classroom (Pellet, 2012). A wiki project can be asynchronous, and from this perspective, it extends language teaching to an effective community of practice. By using wikis, blogs and forums, students can practice the language in a more informal and relaxed environment, at their own pace and with enhanced flexibility.

\subsection{Impact on foreign language competence}

Clearly, wikis mostly rely on the production of written text, so most studies focus on writing and linguistic capabilities improvement in the virtual classroom (e.g. Mak and Coniam, 2008; Li and Zhu, 2017; Aydin and Yildiz, 2014; Hudson, 2018). Hudson (2018) reports that the majority of learners felt wikis useful to advance in the target language, and writing in particular. Specifically, they believed that interactions with more advanced students helped them learn proper grammatical structures or how to approach certain types of writing properly. Moreover, feedback makes students more aware on their mistakes in the foreign language.

Such interactions are crucial for improvements in writing (Wang, 2014), but not only. Sánchez-Gómez (2017) found that the use of wikis contributes to both verbal and non-verbal aspects of language competence, including vocabulary and grammar, as well as culture and discourse competence.

deHaan et al. (2012) reported enhanced foreign language competence, but also greater confidence among their sample of English learners in Japan. Interestingly, the scholars note that even if wiki projects normally do not have an external audience, students perform as if there is such, or saw themselves as the audience.

Less researched yet evident added benefit of interaction in wiki groups is the greater quality of the end result, such as an essay in the target language ( $\mathrm{Li}$ and $\mathrm{Zhu}, 2017)$. Similarly, Pellet (2012) reported that wikis contribute to learners-generated content knowledge. For example, her sample was able not only to improve French writing and linguistic competencies, but also gained factual knowledge about French Sociolinguistics and Modern French. The scholar specifies that such positive outcome very much depends on the tutor's continuous role to provide feedback, to make sure that student's output is of good quality and there is also equal participation.

On the other hand, comprehension and competence can easily be damaged if the wiki tasks are not aligned with individual language level. Most wiki tasks are designed for at least intermediate level learners. However, Kennedy \& Miceli (2013) tested how beginners respond to them. Their survey revealed that only a minority of participants had positive attitudes towards the wiki and found it beneficial for learning outcomes. Problems were stemming from lack of training, but also technical and etiquette problems and the tutor's role.

\section{Raised awareness}

The literature review identified significant pedagogical benefits of wikis for the foreign language tutor. This Web 2.0 tool is practical and user-friendly for both instructors and pupils. Moreover, wikis allow for interactions and foster collaboration and learner-centred pedagogy. Taken together, the opportunities wikis offer have great potential for improving learning outcomes.

However, the interactive and collaborative nature of wikis does not necessarily deliver the desired results. Foreign language learners may feel it unnatural or disturbing to engage in a dialogue with others through this platform. Technical issues often arise, and there might be unequal participation. Even if participants interact and everything goes smoothly, this does not automatically result in true collaboration or accomplished learning objectives.

Despite the theoretical advancements on the topic of wikis' use in foreign language tutoring, the reality is somewhat different. As Reinhardt (2019) points out, most students are not familiar with wikis. They might not be comfortable with the Web 2.0 tool, and need additional guidance to take advantage of the benefits it offers.

Without the strong figure of the tutor, wikis can easily turn into a sloppy practice that is a waste of time and actually demotivates the class. The question then becomes one of what should the teacher do (and not do), when a wikienabled project takes place.

\section{Possible interventions}

When wiki-enabled learning needs to be improved, teachers have a choice: to change themselves or change the students. Clearly, the former, since changing the others is outside one's control. A repeating theme in literature is 
the one of the teacher's role as a key determinant of the wiki project success (e.g. Pellet, 2014; Hudson, 2018; Alghasab, Hardman and Handley, 2019). Research suggests some possible tutor interventions to support students in the wiki context.

In a broader sense, Lund (2004) investigated the role of tutors in web writing. The scholar identified five key roles: 1) pedagogical, or helping through questions and feedback; 2) social-emotional, or encouraging relationships; 3) interactional, or motivating participation and scaffolding; 4) managerial and 5) technical. Likewise, Mangenot and Nissen (2006) observed that their sample of distance learning teachers helps students at three levels: 1) organisational, planning and managing an online task; 2) socio-affective, fostering relationships and community building; 3) socio-cognitive: motivating building on ideas to generate understanding.

Alghasab, Hardman and Handley (2019) specifically examined the teacher's role in wiki-mediated writing activities. Teachers in their observation interacted through moves like setting collaborative rules, praising, guiding writing steps, providing feedback, encouraging individual contributions, editing, monitoring and suggesting sources. Building on previous research, the academics found that a dialogic engagement between the foreign language instructor and students contributes productive talk. On the other hand, directive interventions have a negative impact on interactions and collaboration.

As discussed, other possible ways for improving wikis adoption and its effectiveness to achieve learning objectives is to move the cognitive burden to students, and ask them to train themselves. While this is a less discussed approach in literature, some authors like Wheeler and Wheeler (2009) described such wiki pedagogy. They indeed involved initial training on wikis, before the project starts. For example students were given a document, explaining the nature of wiki-enabled assignments. However, first, their sample was consisted of MSc IT students, who essentially have good level of information technology knowledge. Second, the researchers themselves note that initial training is not sufficient to make the best out of the wiki exercise. They noticed that participants we not fully using the technical capabilities of the platform.

Overall, Wheeler and Wheeler's recommendation (2009) for teachers is to provide guidance throughout the wiki exercise, not only before that. Therefore, it is argued in the present paper that the most effective intervention in wiki pedagogy should come from the teacher, and not from the student.

\section{Conclusion}

This paper discussed wikis from different angles to reach the conclusion that the great interest in this language learning tool is justified by the differentiated results it can deliver. Web 2.0 is not just a buzzword in education, and teachers need to familiarise themselves with its various platforms to make foreign language tutoring more relevant and effective.

Therefore, the present theoretical research resulted in hands-on insights on how wikis can be adopted in language courses. It raised the question of what strategies the tutor can adopt and what pitfalls to avoid, and to a large extent managed to provide a sound basis for future research efforts.

The researcher is now step further in solving the puzzle of how Web 2.0 tools could improve foreign language education, which is a topic of both professional and personal significance. Unfortunately, there were time and access constraints that prevented the author to delve into wiki-related literature in detail.

Nevertheless, several areas of future research has been raised as a result of developing this research paper, including specific impact of variables such as interaction, engagement, motivation, collaboration on learning outcomes in the context of foreign language pedagogy.

\section{References}

1. Alshumaimeri, Y. (2011). The effects of wikis on foreign language students writing performance. Procedia-Social and Behavioral Sciences, 28, 755-763.

2. Arnold, N., Ducate, L., \& Kost, C. (2009). Collaborative writing in wikis: Insights from culture projects in intermediate German classes. Applied Linguistics Faculty Publications and Presentations, 14.

3. Aydin, Z., \& Yildiz, S. (2014). Use of wikis to promote collaborative EFL writing. Language Learning \& Technology, 18(1), 160-180.

4. Biasutti, M. (2017). A comparative analysis of forums and wikis as tools for online collaborative learning. Computers \& Education, 111, 158-171. 
5. Boulos, M. and Wheelert, S. (2007) „, The emerging Web 2.0 social software: An enabling suite of sociable technologies in health and healthcare education. Health Information and Libraries Journal, 24 (1), pp.223.

6. Bradley, L., Lindström, B., \& Rystedt, H. (2010). Rationalities of collaboration for language learning in a wiki. ReCALL, 22(2), 247-264.

7. Chase, D. (2007). Transformative sharing with instant messaging, wikis, interactive maps, and Flickr. Computers in Libraries, 27, 7-8, 52-54, 56.

8. Cowan, W., Herring, S. D., Rich, L. L., \& Wilkes, W. (2009). Collaborate, Engage, and Interact in Online Learning, Successes with Wikis and Synchronous Virtual Classrooms at Athens State University. The 14th Annual Instructional Technology Conference, http://www.athens.edu, March 30 - April 1, 2009, 1-14.

9. DeHaan, J., N. Johnson, N. Yoshimura \& T. Kondo (2012). Wiki and digital video use in strategic interaction-based experiential EFL learning. CALICO Journal, 29(2), 249- 268.

10. Donato, R. (2004) Aspects of collaboration in pedagogical discourse. Annual Review of Applied Linguistics, 24, 284-302.

11. Ducate, L. C., Anderson, L. L., \& Moreno, N. (2011). Wading through the world of wikis: An analysis of three wiki projects. Foreign Language Annals, 44(3), 495-524.

12. Hauck, M., \& Youngs, B. (2008). Telecollaboration in multimodal environments: The impact of task design and learner interaction. Computer Assisted Language Learning, 21(2), 87-124.

13. Huang, T. C. K., \& Huang, C. H. (2010). An integrated decision model for evaluating educational web sites from the fuzzy subjective and objective perspectives. Computers \& Education, 55(2), 616-629.

14. Hudson, J. (2018). Using Wikis for collaborative writing in the ELT classroom. International Journal of Pedagogy and Teacher Education, 2(2), 413-426.

15. Kennedy, C., \& T. Miceli (2013). In piazza online: Exploring the use of wikis with beginner foreign language learners. Computer Assisted Language Learning, 26(5), 389-411.

16. Kessler, G. (2009). Student-initiated attention to form in wiki-based collaborative writing. Language Learning \& Technology, 13(1), 79-95.

17. Levy, M. (2009) WEB 2.0 implications on knowledge management. Journal of knowledge management, 13(1), pp.120-134.

18. Li, M., \& Zhu, W. (2011). Patterns of computer mediated interaction in small writing groups using wiki. Computer Assisted Language Learning, 26(1), 61-82.

19. Li, M., \& Zhu, W. (2017). Good or bad collaborative wiki writing: Exploring links between group interactions and writing products. Journal of Second Language Writing, 35, 38-53.

20. Lund, A. (2008) Wikis: a collective approach to language production. ReCALL, 20(1), 35-54.

21. Lund, K. (2004). Human support in CSCL. In What we know about CSCL (pp. 167-198). Springer, Dordrecht.

22. Mak, B., \& Coniam, D. (2008). Using wikis to enhance and develop writing skills among secondary school students in Hong Kong. System, 36(3), 437-455.

23. Mangenot, F., \& Nissen, E. (2006). Collective activity and tutor involvement in e-learning environments for language teachers and learners. CALICO journal, 601-622.

24. McLeish, J. (1968). The Lecture Method. Cambridge Institute of Education, Cambridge, England.

25. Pellet, S. H. (2012). Wikis for building content knowledge in the foreign language classroom. CALICO Journal, 29(2), 224-248.

26. Reinhardt, J. (2019). Social media in second and foreign language teaching and learning: Blogs, wikis, and social networking. Language Teaching, 52(1), 1-39.

27. Reo, R. (2006). Scaffolding Student Collaboration for Group Wiki Projects. In Mader, S. Using Wikis in Education.

28. Sánchez-Gómez, M. (2017). The Impact of Wikis and Discussion Boards on Learning English as a Second Language. A Mixed Methods Research. Digital Education Review, 32, 35-59.

29. Singel, R. (2005). Are you ready for WEB 2.0?. Available at: http://www.wired.com/sciencel discoveries/news/2005/10/69114 [Accessed 27 February 2021].

30. Vygotsky, L. S. (1978). Mind in society. Cambridge, MA: Harvard University Press

31. Wang, J., Zou, B., Wang, D., \& Xing, M. (2013). Students' perception of a wiki platform and the impact of wiki engagement on intercultural communication. System, 41(2), 245-256.

32. Wang, Y. C. (2014). Using wikis to facilitate interaction and collaboration among EFL learners: A social constructivist approach to language teaching. System, 42, 383-390.

33. Warschauer, M. \& D. Grimes (2007). Audience, authorship, and artifact: The emergent semiotics of Web 2.0. Annual Review of Applied Linguistics, 27, 1-23.

34. Wheeler, S., \& Wheeler, D. (2009). Using wikis to promote quality learning in teacher training. Learning, Media and Technology, 34(1), 1-10. 
35. Wicks, D. (2006). Navigating the Wiki Maze Using a Wiki Tool within a Course Management System. In Mader, S. Using Wikis in Education.

36. Yap, W. L., Neo, M., \& Neo, T. K. (2016). Learner-Centred Teaching Contributes in Promising Results in Improving Learner Understanding and Motivation: A Case Study at Malaysia Tertiary Education. Electronic Journal of e-Learning, 14(4), 266-281. 